\title{
Enabling User Relaying In MCM-NOMA Under Doubly Selective Channels Using Iterative Interference Cancellation Schemes For Wireless loT Networks
}

This paper was downloaded from TechRxiv (https://www.techrxiv.org).

\section{LICENSE}

CC BY 4.0

SUBMISSION DATE / POSTED DATE

01-01-2022 / 05-01-2022

\section{CITATION}

Hamza, Anis Amazigh; Dayoub, lyad; Alouani, Ihsen; Amrouche, Abderrahmane (2022): Enabling User Relaying In MCM-NOMA Under Doubly Selective Channels Using Iterative Interference Cancellation Schemes For Wireless IoT Networks. TechRxiv. Preprint. https://doi.org/10.36227/techrxiv.17710865.v1

$\mathrm{DOI}$ 


\title{
Enabling User Relaying In MCM-NOMA Under Doubly Selective Channels Using Iterative Interference Cancellation Schemes For Wireless loT Networks
}

\author{
Anis Amazigh Hamza ${ }^{1,2, * * *}$, lyad Dayoub ${ }^{2,3^{* *}}$, Ihsen Alouani ${ }^{2,3^{*}}$, and Abderrahmane Amrouche ${ }^{1}$ \\ ${ }^{1}$ Laboratory of Speech Communication and Signal Processing, Faculty of Electronics and Computer Science, University of Science and \\ Technology Houari Boumediene, Bab Ezzouar, 16111, Algiers, Algeria \\ ${ }^{2}$ Polytechnic University Hauts-de-France, CNRS, Univ. Lille, ISEN, Centrale Lille, UMR8520- IEMN - Institut D'Electronique de \\ Microlectronique Et de Nanotechnologie - DOAE - Dpartement Opto-Acousto-Electronique, 59313 Valenciennes, France \\ 3 INSA Hauts-de-France, 59313 Valenciennes, France. \\ * Member, IEEE \\ ** Senior Member, IEEE \\ *** Student Member, IEEE
}

\begin{abstract}
Cell-edge users of the future cellular internet of things (loT) with massive loT sensors can suffer from extremely severe channel conditions, especially under very high-speed scenarios. In this paper, we present a performance improvement method for cell-edge users of multi-carrier modulation (MCM)-based non-orthogonal multiple access (NOMA) downlink systems. To this end, we consider the implementation of cooperative user relaying NOMA (CUR-NOMA) and derive its lower bound E2E-BER under doubly selective channels. In addition, the imperfect successive interference cancellation (SIC) process is analyzed, wherein two interference cancellation schemes are combined to remove the NOMA induced inter-user interference (IUI) and the doubly selective channel induced inter-carrier interference (ICI).
\end{abstract}

Index Terms-Interference, loT, non-orthogonal multiple access, cooperative relaying, doubly selective channels, multi-carrier modulation.

\section{INTRODUCTION}

NOMA has been recognized as a promising multiple access scheme for future wireless networks such as the emerging cellular IoT with massive IoT sensors and devices [1] as it effectively improves the network performance [2]. The majority of papers on NOMA and cooperative communication networks assume a perfect SIC (P-SIC). However, due to hardware limitations and channel estimation errors, SIC is imperfect in most practical cases. In this regard, recent papers are pushing the research ahead by going beyond that assumption. Recently, some works tried to investigate the performance degradation that occurs due to imperfect SIC (I-SIC) [3]-[6]. However, the existing contributions exploring CUR-NOMA mainly focus on single-carrier scenarios. On the other hand, Multi-Carrier Modulation (MCM) based transmission has proven better performances for combating the hostility of wireless channels and providing high data rates. Few studies can be found on MCM-NOMA systems. In [7] a NOMA scheme based on combining the orthogonal frequency division multiple access (OFDMA) with the multi-carrier code division multiple acces (MC-CDMA) is proposed. The resource allocation for MCM-NOMA systems with I-SIC is studied and optimized in [8]. The authors of [9] derived the expressions of the outage probability of MCM-NOMA downlink system.

The common aspect of all the aforementioned studies is that they assume that each subcarrier undergoes quasi-static non-selective flat fading. However, this assumption does not hold in very highspeed scenarios, where the wireless channels are highly doubly selective [10]. Moreover, the high mobility nature of the modern transportation systems leads to more interference and channel estimation errors which affect link reliability and system robustness [11]. The implementation of NOMA with different MCM waveforms under doubly selective scenarios is investigated in [12] wherein an efficient joint interference cancellation scheme is proposed. However, in [12] we considered only the signal processing applied at the level of the cell-center user. In practice, the challenges facing the cell-edge user are more interesting since it experiences poor channel conditions compared to the cell-center user.

To the best of our knowledge, there is a lack of works investigating the performance of cell-edge users of CUR-MCM-NOMA with I-SIC under doubly selective environments. Motivated to develop this treatise, we present in this paper a performance improvement method for cell-edge users of MCM-NOMA systems with I-SIC under doubly selective wireless channels. In this regard, we consider the implementation of a cooperative user relaying scheme, wherein the cell-center user exploits the joint interference cancellation scheme to serve as DF relay and assist the communication between the cell-edge user and the base station (BS). Furthermore, we combine it with an efficient interference cancellation scheme applied by the cell-edge user to reduce the inter-carrier interference (ICI) caused by the doubly selective nature of the relaying link.

\section{SYSTEM MODEL}

We consider a downlink single-cell MCM-based CUR-NOMA network, in which a BS transmits data to a set of users. The set of users is divided into two subsets based on the channel strength of the users, i.e., the set of users with strong channel gains are called cell-center users (CUs), and the set of users with low channel gains are referred to as cell-edge users (EUs). As in [5], we assume that only two users are multiplexed on the same resource block (RB), wherein one is selected from the CUs and the other is selected from the EUs. Different from previous works, we assume all the wireless links to be doubly selective channels. Furthermore, we assume the worst-case scenario for the EU wherein the direct link between the BS and the EU is blocked by some obstacles, and hence it cannot be used to convey the information as it is shown in Fig. 1. The CU is used as a DF relay to assist the communication between the BS and the EU and the MCM-based CUR-NOMA consists of two phases, as described in the following. 


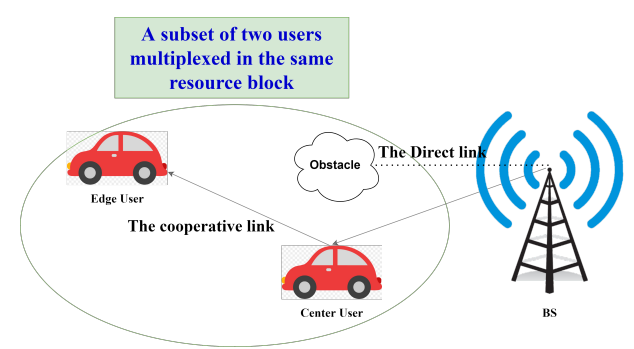

Fig. 1. The system model of the considered very high speed scenario.

During the direct transmission phase, the BS transmits the superposed MCM-NOMA signal to the CU. According to [12] the transmitted signal $s(t)$ consisting of $M$ subcarriers and $K$ timesymbols can be expressed as

$$
\begin{aligned}
s(t) & =\sum_{k=1}^{K} \sum_{m=1}^{M} g_{m, k}(t) x_{m, k} \\
x_{m, k} & =\sqrt{\alpha} x_{E U, m, k}+\sqrt{1-\alpha} x_{C U, m, k}
\end{aligned}
$$

$x_{E U, m, k}, x_{C U, m, k}$ are the transmitted symbols at the $m$ th subcarrier and the $k$ th time symbol of the EU and the CU, respectively. We assume that the powers of the transmitted symbols are normalized such that the average power of the NOMA signal $P_{x}=1 W . \alpha$ denotes the PA coefficient of the EU. $g_{m, k}(t)$ denotes the basis pulses which are, essentially, time and frequency shifted versions of the prototype filter $p_{t x}(t)$ :

$$
g_{m, k}(t)=p_{t x}(t-k T) e^{j 2 \pi m F(t-k T)}
$$

After conducting the sampling process, (1) can be written in a matrix form as [13]

$$
\begin{aligned}
\boldsymbol{s} & =\boldsymbol{G} \boldsymbol{x} \\
\boldsymbol{G} & =\left[\begin{array}{llll}
\boldsymbol{g}_{1,1} & \boldsymbol{g}_{2,1} \ldots \boldsymbol{g}_{M, 1} & \boldsymbol{g}_{1,2} \ldots \boldsymbol{g}_{\boldsymbol{M}, \boldsymbol{K}}
\end{array}\right] \\
\boldsymbol{x} & =\left[\begin{array}{llll}
x_{1,1} & x_{2,1} \ldots x_{\boldsymbol{M}, 1} & x_{1,2} \ldots x_{M, K}
\end{array}\right]^{T}
\end{aligned}
$$

The vector $\boldsymbol{g}_{m, k} \in \mathbb{C}^{N \times 1}$ represents the sampled basis pulses of (3) and builds the transmit matrix $\boldsymbol{G} \in \mathbb{C}^{N \times M K}$ while $\boldsymbol{x} \in \mathbb{C}^{M K \times 1}$ stacks all the transmitted data symbols in one vector. $N$ denotes the number of samples of the whole transmission block. Hence, the demodulated signal at the $\mathrm{CU}$ can be written in a matrix form as

$$
\begin{aligned}
\boldsymbol{y}_{C U} & =\boldsymbol{D}_{C U} \boldsymbol{x}+\boldsymbol{Q}_{C U} \boldsymbol{n}_{C U} \\
\boldsymbol{D}_{C U} & =\boldsymbol{Q}_{C U} \boldsymbol{H}_{C U} \boldsymbol{G}
\end{aligned}
$$

where $\boldsymbol{D}_{C U}$ is called the system transmission matrix (STM), $\boldsymbol{Q}_{C U} \in$ $\mathbb{C}^{M K \times N}$ denotes the demodulation matrix, the Gaussian distributed noise at the $\mathrm{CU}$ is denoted by $\boldsymbol{n}_{C U} \in \mathbb{C}^{N \times 1}$, and $\boldsymbol{H}_{C U} \in \mathbb{C}^{N \times N}$ is the doubly-selective channel convolution matrix at the CU. As stated before, the CU performs I-SIC, where it first detects the EU data while considering $x_{C U}$ as interference.

$$
\hat{\boldsymbol{x}}_{E C}=\mathcal{M}\left(\frac{1}{\sqrt{\alpha}} \boldsymbol{A}_{E C} \boldsymbol{y}_{C U}\right)
$$

where $\hat{\boldsymbol{x}}_{E C}$ denotes the data of the EU detected during the first step of the SIC and $\mathcal{M}($.$) is the quantization operator, that is, nearest$ neighbor detection while $\boldsymbol{A}_{E C}$ is the adopted equalizer that estimates the EU data at the CU. To detect the data of the CU, $\hat{\boldsymbol{x}}_{E C}$ will be removed from the received signal as follows

$$
\hat{\boldsymbol{x}}_{C U}=\mathcal{M}\left(\frac{1}{\sqrt{1-\alpha}} \boldsymbol{A}_{C U}\left(\boldsymbol{y}_{C U}-\hat{\boldsymbol{D}}_{C U} \sqrt{\alpha} \hat{\boldsymbol{x}}_{E C}\right)\right)
$$

where $\boldsymbol{A}_{C U}$ denotes the adopted equalizer that estimates the data of the CU and the hat ${ }^{1}$ in $\hat{\boldsymbol{D}}_{C U}$ denotes the estimated STM. During the cooperative phase, the $\mathrm{CU}$ regenerates the data of the EU detected in the first step of the I-SIC process and forwards it to the EU with an average power $P_{\hat{x}_{E C}}=P_{x}$. Similar to the direct phase the received signal at the EU after the sampling is given by

$$
\begin{aligned}
\boldsymbol{y}_{E U} & =\boldsymbol{D}_{E U} \hat{\boldsymbol{x}}_{E C}+\boldsymbol{Q}_{E U} \boldsymbol{n}_{E U} \\
\boldsymbol{D}_{E U} & =\boldsymbol{Q}_{E U} \boldsymbol{H}_{E U} \boldsymbol{G}_{E U}
\end{aligned}
$$

The EU can then detect its signal directly as follows

$$
\hat{\boldsymbol{x}}_{E U}=\mathcal{M}\left(\boldsymbol{A}_{E U} \boldsymbol{y}_{E U}\right)
$$

Note that we assume both wireless links $\boldsymbol{H}_{C U}$ and $\boldsymbol{H}_{E U}$ to be doubly selective. Therefore, the received power will be spread across the non-diagonal elements of the STM matrices, and hence the use of a simple one-tap equalizer ${ }^{2} \boldsymbol{A}=\operatorname{diag}(\hat{\boldsymbol{D}})^{-1}$, where $\operatorname{diag}(\hat{\boldsymbol{D}})$ gives the diagonal matrix of $\hat{D}$, might not be sufficient.

\section{EQUALIZATION AND INTERFERENCE CANCELLATION}

To reduce the interference during the direct transmission phase, the CU will exploit the joint iterative algorithm which consist of the following steps.

Initially, the CU detects the data of the EU and then his own data by considering a one-tap equalizer at (9) and (10), respectively. After that, it starts to remove the interference at each iteration as follows

$$
\begin{aligned}
& \overline{\boldsymbol{y}}_{E}^{(i)}=\boldsymbol{y}_{C U}-\underbrace{\operatorname{ndiag}\left(\hat{\boldsymbol{D}}_{C U}\right) \sqrt{\alpha} \hat{\boldsymbol{x}}_{E C}^{(i-1)}}_{\boldsymbol{I} \boldsymbol{I} \boldsymbol{I}}-\underbrace{\hat{\boldsymbol{D}}_{C U} \sqrt{1-\alpha} \hat{\boldsymbol{x}}_{C U}^{(i-1)}}_{I U \boldsymbol{I}} \\
& \hat{\boldsymbol{x}}_{E C}^{(i)}=\mathcal{M}\left(\frac{1}{\sqrt{\alpha}} \operatorname{diag}\left(\hat{\boldsymbol{D}}_{C U}\right)^{-1} \overline{\boldsymbol{y}}_{E}^{(i)}\right) \\
& \overline{\boldsymbol{y}}_{C}^{(i)}=\boldsymbol{y}_{C U}-\underbrace{\operatorname{ndiag}\left(\hat{\boldsymbol{D}}_{C U}\right) \sqrt{1-\alpha} \hat{\boldsymbol{x}}_{C U}^{(i-1)}}_{I C I}-\underbrace{\hat{\boldsymbol{D}}_{C U} \sqrt{\alpha} \hat{\boldsymbol{x}}_{E C}^{(i)}}_{I U I} \\
& \hat{\boldsymbol{x}}_{C U}^{(i)}=\mathcal{M}\left(\frac{1}{\sqrt{1-\alpha}} \operatorname{diag}\left(\hat{\boldsymbol{D}}_{C U}\right)^{-1} \overline{\boldsymbol{y}}_{C}^{(i)}\right)
\end{aligned}
$$

where $\operatorname{ndiag}(\hat{\boldsymbol{D}})$ zeros the diagonal elements of $\hat{\boldsymbol{D}}$ while keeping the off-diagonal elements. The term $\overline{\boldsymbol{y}}_{E}^{(i)}$ in (14) denotes the result of the $i$ th iteration which is used to detect the data of the EU at the $\mathrm{CU}$, whereas $\overline{\boldsymbol{y}}_{C}^{(i)}$ in (16) is used to detect the data of the CU. Note that in (14) the $(i-1)$ th detected data of the EU, is used to remove the off-diagonal induce ICI while the $(i-1)$ th detected data of the $\mathrm{CU}$ is used to cancel the NOMA induced IUI. (16) follows the same principle as (14) (see Algorithm 1 at top of the next page).

After $L_{1}$ iterations the $\mathrm{CU}$ is ready to transmit the detected $\hat{\boldsymbol{x}}_{E C}^{\left(L_{1}\right)}$ to the edge user. However, due to the doubly selective nature of the cooperative link, new ICI will occur at the received signal of the EU. The received signal at the EU can be written in a matrix form exactly as in (11) by just replacing $\hat{\boldsymbol{x}}_{E C}$ by $\hat{\boldsymbol{x}}_{E C}^{\left(L_{1}\right)}$ since in here, we assume that the $\mathrm{CU}$ has already applied the joint iterative algorithm before starting the cooperative phase. To cancel the new ICI we propose a simple, yet effective, interference cancellation scheme. Similar to

${ }^{1}$ As mentioned in [14], it is computationally more efficient to directly estimate $\hat{\boldsymbol{D}}$ compared to the channel transfer function $\hat{\boldsymbol{H}}$.

${ }^{2}$ Note that a one-tap equalizer requires $O(M K)$ computational complexity since only the diagonal of $D \in \mathbb{C}^{M K \times M K}$ is inverted. 


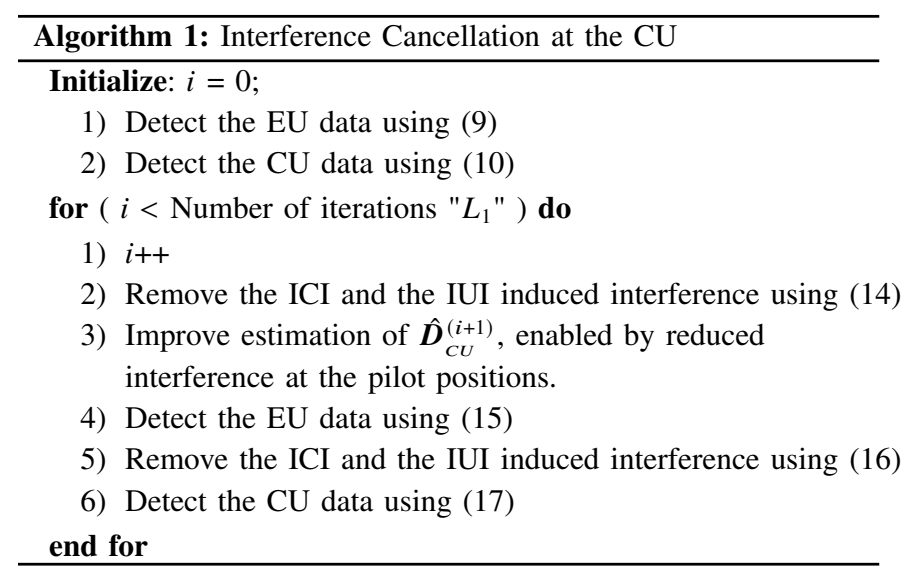

the one suggested in [15] for OFDM waveforms, the ICI is canceled as follows

$$
\begin{aligned}
& \overline{\boldsymbol{y}}_{E U}^{(i)}=\boldsymbol{y}_{E U}-\underbrace{\operatorname{adiag}\left(\hat{\boldsymbol{D}}_{E U}\right) \hat{\boldsymbol{x}}_{E U}^{(i-1)}}_{I C \boldsymbol{I}} \\
& \hat{\boldsymbol{x}}_{E U}^{(i)}=\mathcal{M}\left(\operatorname{diag}\left(\hat{\boldsymbol{D}}_{E U}\right)^{-1} \overline{\boldsymbol{y}}_{E U}^{(i)}\right)
\end{aligned}
$$

First, the edge user detects its signal using (13) adopting the one-tap equalizer $\boldsymbol{A}_{E U}=\operatorname{diag}\left(\hat{\boldsymbol{D}}_{E U}\right)^{-1}$. Then, it applies the iterative scheme using (18) and (19) (see Algorithm 2 below).

The most computationally expensive part of each algorithm is the vector-matrix multiplication used in the interference calculations (see (14), (16), and (18)). Therefore, the computational complexity of each algorithm is $O\left((M K)^{2}\right)$ per iteration ${ }^{3}$ which is very low compared to the full block MMSE that requires $O\left((2 M K)^{3}\right)$ operations as it has to invert a matrix of a size of $2 M K \times 2 M K$.

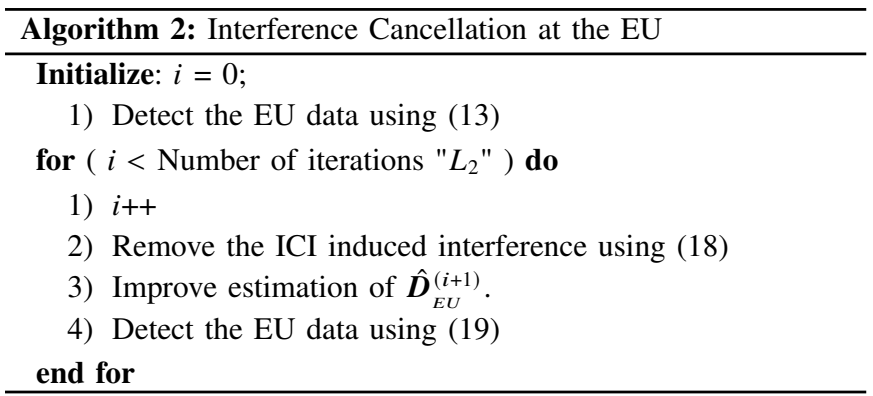

\section{THE END-TO-END BIT ERROR RATE}

An erroneous detection can occur either in the direct transmission phase or the cooperative phase. Thus, the end-to-end bit error rate (E2E-BER) can be expressed as:

$$
\text { E2E-BER }=1-\left(1-P_{\text {SIC }}\right)\left(1-P_{\text {coop }}\right)
$$

Where $P_{\text {SIC }}$ denotes the probability that $x_{2}$ is detected erroneously during the SIC process of the direct phase, and $P_{\text {coop }}$ denotes the probability that the EU detects erroneously the relaying link signal during the cooperative phase. The lower bound E2E-BER that describes the best performance that could be achieved under doubly selective channels would be the E2E-BER of the theoretical doubly

${ }^{3}$ Only 4 iterations are needed for each algorithm (see Fig. 3).
TABLE 1. $f(x)$ for different modulation orders

\begin{tabular}{lll}
\hline$f(x)$ & modulation order & waveform \\
\hline$f(x)=\frac{1}{2}-\frac{1}{2 \sqrt{1+\frac{2}{x}}}$ & 4-QAM & FBMC-OQAM \\
$f(x)=\frac{1}{2}-\frac{3}{8 \sqrt{1+\frac{10}{x}}}-\frac{6}{8 \sqrt{9+\frac{10}{x}}}+\frac{5}{8 \sqrt{25+\frac{10}{x}}}$ & 16-QAM & FBMC-OQAM
\end{tabular}

TABLE 2. Simulation parameters

\begin{tabular}{ll}
\hline Parameter & value \\
\hline Number of symbols & 30 \\
Number of subcarriers & 24 \\
Number of auxilary symbols (per pilot) & 4 \\
Pilot-to-data power offset $\left(\frac{P_{p}}{P_{D}}\right)$ & 4.685 \\
Pilot overhead & $11.1 \%$ \\
Subcarrier spacing & $15 \mathrm{kHz}$ \\
Carrier frequency & $2.5 \mathrm{GHz}$ \\
Waveform & FBMC-OQAM \\
Modulation order & 4 \\
Channel gain $G_{1}$ & 0.8 \\
Channel gain $G_{2}$ & 0.6 \\
CU's speed & $200 \mathrm{~km} / \mathrm{h}$ \\
EU's speed & $100 \mathrm{~km} / \mathrm{h}$ \\
PA coeffecient $\alpha$ & 0.9
\end{tabular}

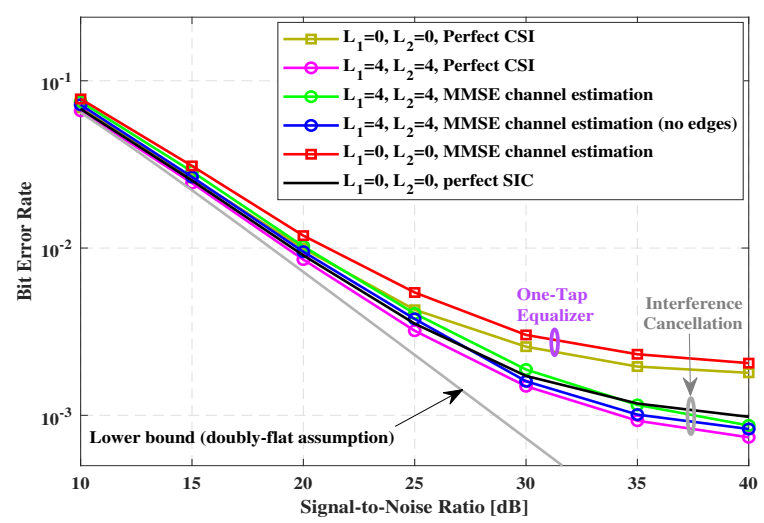

Fig. 2. The E2E-BER vs SNR.

flat channels with zero NOMA induced IUI. Hence, using [13, Eq. (15)-(18)], it can be shown that the lower bound E2E-BER that can be achieved by CUR-MCM-NOMA system is given by

$$
\mathrm{E}_{2} \mathrm{E}_{-\mathrm{BER}}^{\mathrm{min}}=1-\left(1-f\left(\frac{2 G_{1} P_{x}}{P_{n}}\right)\right)\left(1-f\left(\frac{2 G_{2} P_{x}}{P_{n}}\right)\right)
$$

Where $f(x)$ is given in Table $1, G_{1}$ denotes the average channel gain of $H_{C U}, G_{2}$ denotes the average channel gain of $H_{E U}$ and $P_{n}$ is the average noise power.

\section{NUMERICAL RESULTS}

In this section, we simulate the performance of the proposed CUR-MCM-NOMA system while taking the performance of the PSIC with perfect channel state information (CSI) as a benchmark for comparison. In all scenarios, we conduct Monte Carlo simulations with 1500 repetitions while considering a channel model with Vehicular-A power delay profile and Jakes Doppler model. Unless stated otherwise, we assume imperfect CSI and consider the auxiliary symbol channel estimation method [14] with the simulation parameters shown in Table 2. Fig. 2 shows the E2E-BER over SNR for CUR-MCMNOMA. It can be seen that our proposed scheme performs close to 


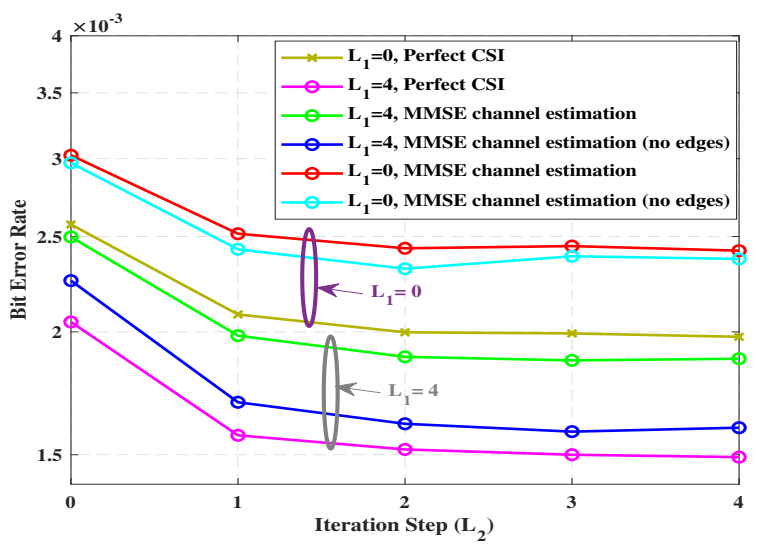

Fig. 3. The E2E-BER vs Number of Iterations $L_{2}$ for $\mathrm{SNR}=30 \mathrm{~dB}$.
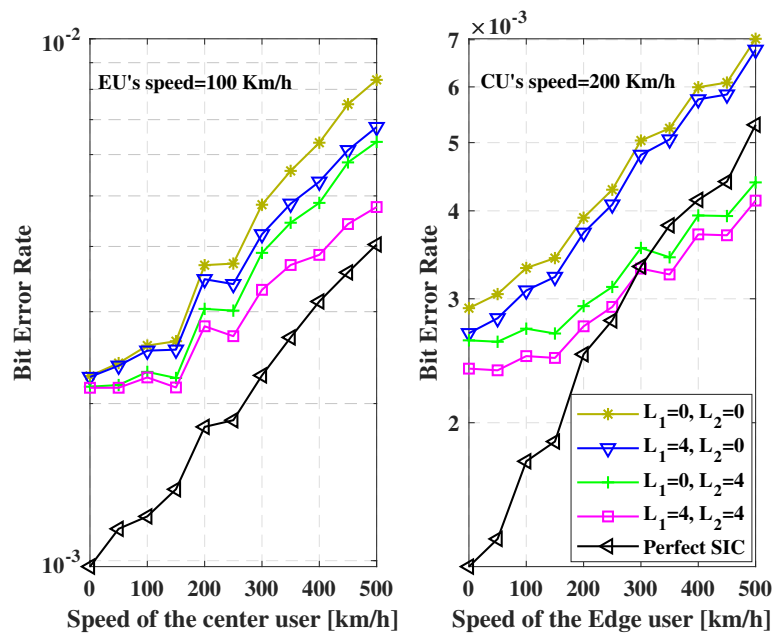

Fig. 4. The E2E-BER vs the speed of each user for $\mathrm{SNR}=30 \mathrm{~dB}$.

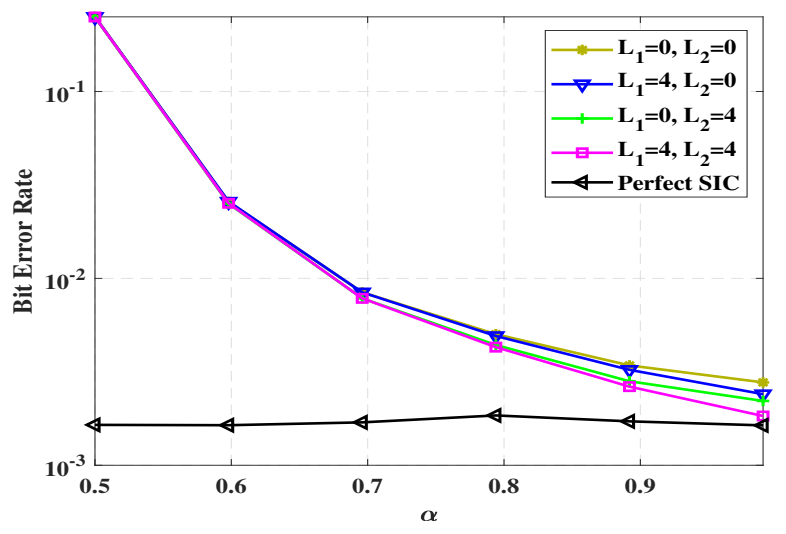

Fig. 5. The E2E-BER vs the PA coefficient $\alpha$ for SNR $=30 \mathrm{~dB}$.

the $\mathrm{P}-\mathrm{SIC}$ and can even outperform the $\mathrm{P}$-SIC in the high-SNR regime $(\mathrm{SNR} \approx 35 \mathrm{~dB})$ or when perfect CSI is provided. Because our scheme reduces the ICI and IUI, whereas the P-SIC cancels only the IUI and keeps the ICI. Another advantage of the proposed iterative algorithms is their fast convergence. This fact is presented in Fig. 3, where we can note how after 3 to 4 iterations both algorithms will converge. One can also note that when both algorithms are combined $\left(L_{1}=4\right)$, the performance gain is significantly increased even when imperfect CSI is assumed. Fig. 4 shows the E2E-BER versus the speed of each user for CUR-MCM-NOMA with perfect CSI. Interestingly, we note that when the EU's speed is very high $\left(V_{E U} \geq 300 \mathrm{~km} / \mathrm{h}\right)$, our proposed scheme $\left(L_{1}=4, L_{2}=4\right)$ outperforms the P-SIC case. Moreover, when the speed of the $\mathrm{CU}$ is very high, the performance of the proposed scheme is very close to that of the P-SIC. Finally, It is worth noting that as with any NOMA-based system, appropriate PA coefficient selection is very crucial, as shown in Fig. 5.

\section{CONCLUSION}

This paper presented a performance improvement method for EUs under very high-speed mobility using cooperative MCM-NOMA technology combined with two efficient interference cancellation schemes. The obtained results have shown that the introduced scheme provides a significant performance improvement while maintaining a fast convergence rate, hence low complexity.

\section{REFERENCES}

[1] L. Chen, B. Hu, G. Xu, and S. Chen, "Energy-Efficient Power Allocation and Splitting for mmWave Beamspace MIMO-NOMA With SWIPT," IEEE Sensors Journal, vol. 21, no. 14, pp. 16381-16394, 2021.

[2] O. Maraqa, A. S. Rajasekaran, S. Al-Ahmadi, H. Yanikomeroglu, and S. M. Sait, "A survey of rate-optimal power domain noma with enabling technologies of future wireless networks," IEEE Communications Surveys Tutorials, vol. 22, no. 4, pp. 2192-2235, 2020

[3] F. Kara and H. Kaya, "On the error performance of cooperative-NOMA with statistical CSIT," IEEE Communications Letters, vol. 23, no. 1, pp. 128-131, 2019.

[4] M. W. Akhtar, S. A. Hassan, S. Saleem, and H. Jung, "STBC-aided cooperative NOMA with timing offsets, imperfect successive interference cancellation, and imperfect channel state information," IEEE Transactions on Vehicular Technology, pp. 1-1, 2020.

[5] L. Zhang, J. Liu, M. Xiao, G. Wu, Y. Liang, and S. Li, "Performance analysis and optimization in downlink NOMA systems with cooperative full-duplex relaying," IEEE Journal on Selected Areas in Communications, vol. 35, no. 10, pp. 2398-2412, 2017.

[6] A. A. Hamza, I. Dayoub, I. Alouani, and A. Amrouche, "On the error rate performance of full-duplex cooperative NOMA in wireless networks," IEEE Transactions on Communications, pp. 1-1, 2021.

[7] A. Maatouk, E. Çalışkan, M. Koca, M. Assaad, G. Gui, and H. Sari, "Frequencydomain NOMA with two sets of orthogonal signal waveforms," IEEE Communications Letters, vol. 22, no. 5, pp. 906-909, 2018.

[8] J. Chen, L. Zhang, Y. Liang, and S. Ma, "Optimal resource allocation for multicarrier NOMA in short packet communications," IEEE Transactions on Vehicular Technology, vol. 69, no. 2, pp. 2141-2156, 2020

[9] S. Li, M. Derakhshani, S. Lambotharan, and L. Hanzo, "Outage Probability Analysis for the Multi-Carrier NOMA Downlink Relying on Statistical CSI," IEEE Transactions on Communications, vol. 68, no. 6, pp. 3572-3587, 2020.

[10] M. Saideh, I. Dayoub, and M. Berbineau, "Efficient Equalization for FBMC-OQAM Under Doubly Selective Channel Estimation Errors," IEEE Communications Letters, vol. 23, no. 5, pp. 863-866, 2019.

[11] A. Alalewi, I. Dayoub, and S. Cherkaoui, "On 5G-V2X use cases and enabling technologies: A comprehensive survey," IEEE Access, vol. 9, pp. 107 710-107737, 2021.

[12] M. Saideh, Y. Alsaba, I. Dayoub, and M. Berbineau, "Joint interference cancellation for multi-carrier modulation-based non-orthogonal multiple access," IEEE Communications Letters, vol. 23, no. 11, pp. 2114-2117, 2019.

[13] R. Nissel and M. Rupp, "OFDM and FBMC-OQAM in Doubly-Selective Channels: Calculating the Bit Error Probability," IEEE Communications Letters, vol. 21, no. 6, pp. 1297-1300, 2017.

[14] R. Nissel, F. Ademaj, and M. Rupp, "Doubly-selective channel estimation in FBMC-OQAM and OFDM systems," in 2018 IEEE 88th Vehicular Technology Conference (VTC-Fall), 2018, pp. 1-5.

[15] A. F. Molisch, M. Toeltsch, and S. Vermani, "Iterative methods for cancellation of intercarrier interference in OFDM systems," IEEE Transactions on Vehicular Technology, vol. 56, no. 4, pp. 2158-2167, 2007. 